\title{
The Results of the Combine Treatment of Patients with Liver Bilobar Metastases from Colorectal Cancer Using Radiofrequency Ablation
}

\author{
Oleg Igorevich Kaganov ${ }^{1}$ (D) Sergei Vasilevich Kozlov ${ }^{1} \cdot$ Andrei Evgenyevich Orlov $^{2} \cdot$ Nikita Vyacheslavovich Blinov $^{1}$
}

Received: 15 February 2017 / Accepted: 28 March 2018 / Published online: 9 April 2018

(C) Indian Association of Surgical Oncology 2018

\begin{abstract}
Colorectal cancer (CRC) is the third most common cancer worldwide. The mortality from CRC remains very high. The main cause of such a high mortality is a disseminate process with the appearance of distant metastases. In this regard, the treatment of metastatic lesions is recognized as an important trend in modern oncology. The program of study included 176 patients with colorectal cancer after primary tumor removal with the malignant progression-multiple (more than 4) bilobar liver metastases. The research was organized in Samara Regional Oncology Centre from 2001 to 2014. By the treatment method, patients were divided into two groups. Main group got the combined (chemotherapy + radiofrequency ablation (RFA)) treatment $(n=98)$. In control group, only chemotherapy was applied $(n=78)$. One-, two-, and three-year OS were 73.5, 25.1, and 7.2\% in the main group and 39.6, 6.3, and 2.1\% in the control group. The RFA application allowed us to reach the index of 4-year survival $1.8 \%$ in the main group, while we received only 2.1 of 3-year survival in the control group. The OS median reached 18 months in the main group and 11 months in the control group. So, the OS curves in two comparing groups were significantly different according to statistics (log-rank test 3.77, $p=0.000$ ). The application of RFA in combination with chemotherapy in the treatment of bilobar metastasis colorectal cancer allows to improve the performance of disease-free survival and overall survival significantly, compared with the group of patients who received only chemotherapy.
\end{abstract}

Keywords Colorectal cancer $\cdot$ Liver bilobar metastases $\cdot$ Radiofrequency ablation

\section{Introduction}

Colon cancer is a current problem of modern oncology [1]. There are about one million of new cases of colorectal cancer

Oleg Igorevich Kaganov oleg.kaganov@inbox.ru

Sergei Vasilevich Kozlov kozlovsv@samaraonko.ru

Andrei Evgenyevich Orlov orlovae@samaraonko.ru

Nikita Vyacheslavovich Blinov nik261094@mail.ru

1 Oncology Department, Samara State Medical University, Samara, Russian Federation

2 Samara Regional Oncology Centre, Samara, Russian Federation
(CRC) identified in the world each year. And more than 50 thousand of them in Russia [2].

The mortality from CRC remains very high. The main cause of such a high mortality is a disseminate process with the appearance of distant metastases. The liver is a major target organ on the hematogenous way to spread secondary neoplasms [3]. Synchronous metastases are diagnosed in 25$35 \%$ cases; dispensary observation revealed about $50-70 \%$ of patients with metastases after the removal of the primary colon tumor in different periods after surgery [4]. In this regard, the treatment of metastatic lesions is recognized as an important trend in modern oncology, which aims to increase lifetime and to improve the quality of life [5].

According to Gleisner A.L. et al. (2008), chemotherapy without surgery slightly increases lifetime of patients with metastatic colorectal cancer; the median of overall survival (OS) follows up to 12-15 months. The reason of this situation is a marked resistance of mutated malignant cells as the result 
of chemotherapy [6]. Thereby, surgical technique is the "gold" standard in the combined treatment of metastatic lesions [7].

Unfortunately, the possibility of CRC metastases' resection does not exceed 15-20\% [8]. This is due to the fact that in most cases, multiple bilobar metastases of both liver lobes are diagnosed. Such spread of the malignant process is the indication for extended liver resection accompanied by a high number of complications and lethality [9, 10]. All that makes surgeons use new types of minimally invasive, organpreserving treatment of patients with bilobar metastatic colorectal cancer in the liver. One of such methods is radiofrequency ablation (RFA) [11].

Originally, RFA was considered only as a palliative procedure, but in clinical recommendations of the European Society for Medical Oncology (ESMO, 2009), it was noted that the results of treatment of the combination of chemotherapy and RFA are under study and are considered as an alternative to surgical treatment of metastatic colorectal cancer; it confirms the topicality of the study carried out [12]. According to different scientists, there is no united standpoint about the place of thermoablation in the combined treatment $[8,13]$.

The aim of this work is to determine the place of RFA in the combined treatment of liver bilobar metastatic process of colorectal cancer.

\section{Materials}

The program of study included 176 patients with colorectal cancer after removal of the primary tumor with the malignant progression - multiple (more than 4) bilobar liver metastases. The research was organized in Samara Regional Oncology Centre from 2001 to 2014. By the treatment method, patients were divided into two groups. Main group got the combined (chemotherapy + RFA) treatment $(n=98)$. In control group, only chemotherapy was applied $(n=78)$.

After the removal of the primary tumor, clinical diagnosis rTNM system was exhibited (Table 1).

Table 1 TNM stage of patients in groups $1 \mathrm{a}$ and $1 \mathrm{~b}$ with colon and rectum cancer

\begin{tabular}{lllllll}
\hline TNM stage & & \multicolumn{2}{c}{ Control $(n=78)$} & & \multicolumn{2}{c}{ Main $(n=98)$} \\
\cline { 3 - 4 } \cline { 6 - 7 } \cline { 6 - 7 } & & $n$ & $\%$ & & $n$ & $\%$ \\
\hline Stage 1 & T1-2 N0 M0 & 10 & 12.8 & & 15 & 15.3 \\
Stage 2 & T3-4 N0 M0 & 60 & 76.9 & & 72 & 73.5 \\
Stage 3 & T1-4 N1-3 M0 & 8 & 10.3 & & 11 & 11.2 \\
Total & & 78 & 100 & & 98 & 100 \\
\hline
\end{tabular}

Pirson criterion $0.999695 ; p=0.8$
In the analyzed groups, there was no colorectal cancer on the stage 4 , since the distant metastases were not detected during the removal of the primary tumor. The most frequent stage in both groups was stage 2 (main -72 , control-60 patients). In the postoperative period, chemotherapy and radiation therapy were not indicated for patients with stage 1 and 2 CRC [113], $11(11.2 \%)$ in the main group and $8(10.3 \%)$ patients in the control group had stage 3 of the prevalence process, which was the indication for chemotherapy in the adjuvant setting, but patients refused this treatment. Thus, research groups got only surgical treatment of primary tumors of the colon.

The average age of the patients in groups was 57, $04 \pm$ 7.67 , and $57.53 \pm 8.22(p=0.68)$. The progression of the process (appearance of distant metastases) was detected on the average by $14.72 \pm 5.85$ months in the main group and by $14.15 \pm 5.91$ months $(p=0.52)$ in the control group after surgery on the colon.

We used CT with contrast enhancement of the abdominal cavity to refine the prevalence of metastatic disease. For verification of secondary neoplasms, needle aspiration biopsy was performed. In all cases cytology result was adenocarcinoma.

The common number of the revealed metastatic node liver was 363 in the control group and 461 in the main group, the average number $-4.05 \pm 1.30$ and $4.01 \pm 1.44$ $(p=0.92)$ and the size $-2.17 \pm 0.58 \mathrm{~cm}$ and $2.14 \pm 0.60$ $(p=0.66)$ (Tables 2 and 3).

Multiple bilobar metastases are contraindications for surgical treatment because of the high activity of prevalence process. Metastases less than $3 \mathrm{~cm}$ were diagnosed in the control and main groups $297(81.8 \%)$ and $372(80.7 \%)$, more than $3 \mathrm{~cm}-66(18.2 \%)$ and $89(19.3 \%)(p=0.93$ и $p=0.96)$.

For complex evaluation of the prevalence of metastasis process, we applied the method used in the RECIST criteria: the sums of the maximum diameter of metastases are counted for each patient; in the main group, it was $8.33 \pm 2.30 \mathrm{~cm}$ and $8.42 \pm 2.65 \mathrm{~cm}(p=0.84)$ for control group.

Thus, the patients in the treatment groups had the similar number and size of the liver metastases from colorectal cancer.

Table 2 The number of CRR liver metastases in the control and main groups

\begin{tabular}{llllll}
\hline $\begin{array}{l}\text { Number of liver } \\
\text { metastases }\end{array}$ & \multicolumn{2}{l}{ Control group $(n=78)$} & & \multicolumn{2}{c}{ Main group $(n=98)$} \\
\cline { 2 - 3 } & $n$ & $\%$ & & $n$ & $\%$ \\
\hline 4 & 40 & 51.3 & & 48 & 49 \\
5 & 25 & 32.1 & & 31 & 31.6 \\
6 & 13 & 16.6 & & 19 & 19.4 \\
Total & 78 & 100 & & 98 & 100 \\
\hline
\end{tabular}

Pirson criterion $0.9993822 ; p=0.6$ 
Table 3 The sizes of CRC liver metastases in the control and main groups

\begin{tabular}{llllll}
\hline \multirow{2}{*}{$\begin{array}{l}\text { Size of liver } \\
\text { metastases }\end{array}$} & \multicolumn{2}{l}{ Control group $(n=363)$} & & \multicolumn{2}{c}{ Main group $(n=461)$} \\
\cline { 2 - 3 } \cline { 5 - 6 } \cline { 5 - 6 } & $n$ & $\%$ & & $n$ & $\%$ \\
\hline Less than $2 \mathrm{~cm}$ & 147 & 40.5 & & 174 & 37.7 \\
$2-3 \mathrm{~cm}$ & 150 & 41.3 & & 198 & 43.0 \\
More than $3 \mathrm{~cm}$ & 66 & 18.2 & & 89 & 19.3 \\
Total & 363 & 100 & & 461 & 100 \\
\hline
\end{tabular}

Pirson criterion $0.9839150 ; p=0.6$

At the first step for the patients from both groups, systemic chemotherapy was applied with 3 weeks interval for the drug combination FOLFOX and FOLFIRI. After each three courses of chemotherapy, control CT of the abdominal cavity was led to estimate treatment efficiency. The results of the performed treatment were assessed with the RECIST criteria. Dynamics of the size and number of metastases were measured by the results of treatment: full answer - the disappearance of all the target centers; the partial response-reduction of at least $30 \%$ of the sum of the largest diameter of the measured metastases from the beginning of treatment; progression of the disease-increase of more than $20 \%$ of the sum of the largest diameter; stabilization - the absence of significant reduction in the sum of the largest diameters.

If the disease was on the stabilization stage after the third course of chemotherapy in the main group, we added RFA for liver metastasis in control group patients who got only chemotherapy.

The main criteria of evaluating the results of treatment were disease-free and overall survival (OS). Overall survival is a term that denotes the chances of staying alive for a group of individuals during the research period. Disease-free survival (DFS) denotes the chances of staying free of relapse CRC liver metastases after a particular treatment for a group of individuals. The overall survival median is the time, when $50 \%$ of the observed patients die. The disease-free survival median is the time, when the cancer progression process was detected in $50 \%$ of patients.

\section{Results}

The results of DFS in the main and control groups are shown in Fig. 1.

The results of DFS in the main group was $13.5 \%$, in control- $10.2 \%$. Using RFA in the main group leads to $3.4 \%$ of $2-$ year DFS, while in the control group, 2-year DFS was 0; DFS median in the main group was higher than that in the control group; it was 9 and 6 months. The difference of DFS curves in the research groups was statistically significant (long-range criteria $2.12, p=0.05)$.
Thereby, the use of RFA in combined treatment helped to improve the results of DFS in patients with CRC multiple bilobar liver metastases, comparing to patients who got only chemotherapy.

According to the CT of the abdomen in the first year of observation after RFA, relapse and progression of the disease were not revealed in 26 patients. Progression of the disease as the form of a relapse after RFA effect and appearance of new metastases was diagnosed in 67 patients; $42(62.7 \%)$ of them had multiple metastases, or their sizes were more than $3 \mathrm{~cm}$ in diameter. Upon further observation in all research groups, the progression of the disease was identified as the form of the appearance of new metastases that was the indication for changing drug combination FOLFOX/FOLFIRI in the main group in $12(13 \%)$ in the control group - in $8(9 \%)$ patients. Stabilization of the metastatic process during the second-line chemotherapy was achieved in the main group in 7 patients; they also got second RFA. Chemoembolization of liver by spheres with doxorubicin was held to 15 patients in the main group and to 16 patients in the control group, which stabilized the progression in 2 and 3 patients. For the rest of the patients in the research groups, special treatment was not indicated, because they got symptomatic treatment in the local polyclinic.

The overall survival rates in the main and research groups are shown in Fig. 2.

The One-, two-, and three-year Overal Survival rate were $73.5,25.1$, and $7.2 \%$ in the main group and 39.6, 6.3, 2.1\% in the control group. The RFA application allowed us to reach the index of 4-year survival $1.8 \%$ in the main group, while we received only 2.1 of 3 -year survival in the control group.

The OS median reached 18 months in the main group and 11 months in the control group. So, the OS curves in two comparing groups were significantly different according to statistics (log-rank test 3.77, $p=0.000$ ).

\section{Discussion}

The treatment of patients with bilobar liver metastases of CRC identified at different periods of time after complete resection of colon tumors is the pressing issue of oncology, as isolated liver metastases are often the only manifestation of the disease progression [14]. The aim of the surgical method in the combined treatment of patients with metastatic colorectal cancer is the reduction of the tumor mass that effects on the success of the application in the subsequent chemotherapy [15].

The main reason for refusal from surgery in cases of CRC with liver metastases is the technical impossibility of all lesion removal (operation R0) but maintaining a sufficient amount of the remaining functional parenchymal organ (at least 25-30\% of the total), which is the most typical for bilobar lesions [9, 16]. The performance of advanced liver resections in patients 
Fig. 1 DFS (Kaplan-Meier) in the main and control groups with multiple bilobar liver metastases of CRC

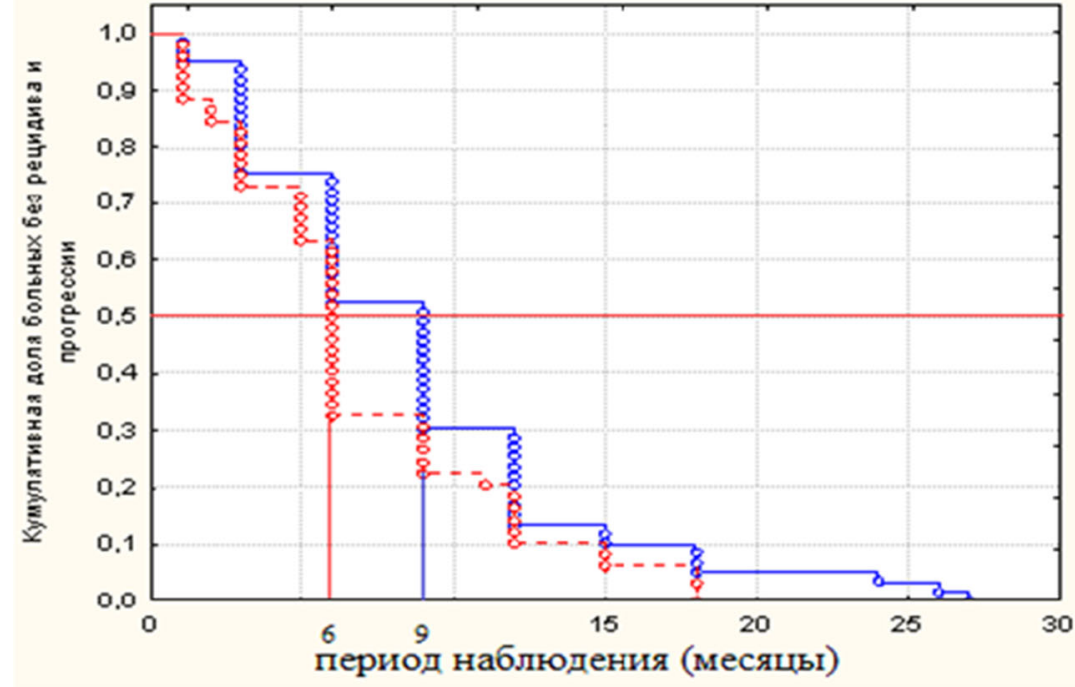

Main group

Control group

with bilobar defeat is connected with a high incidence of intraand postoperative complications and low rates of disease-free and overall survival [13]. According to Younes R.N. et al. (1999), Mynster T. (2000), and Jamagin W.R. et al. (2002), survival indices are linked with the volume of bleeding and the number of complications that effects on the prolongation of immunosuppression which may promote proliferation of tumor cells. Insufficient operation of liver parenchyma causes the release of large number of growth factors which is necessary for start of the regeneration process that also contributes to the progression of malignant disease [6].

The gradual shift in surgeon's emphases from the mechanistic perception of liver tumors as the focus to be removed, to the understanding of it as one of the manifestations of systemic cancer process, forces the surgeon to think about the correlation of the benefit from the removal of the tumor and the harm from immunosuppression caused by surgery (Weese J.L. et al. 1986; Shakhar G. et al. 2003). In some cases, a minimally invasive
Fig. 2 OS (Kaplan-Meier) in the main and control groups with multiple bilobar liver metastases of CRC

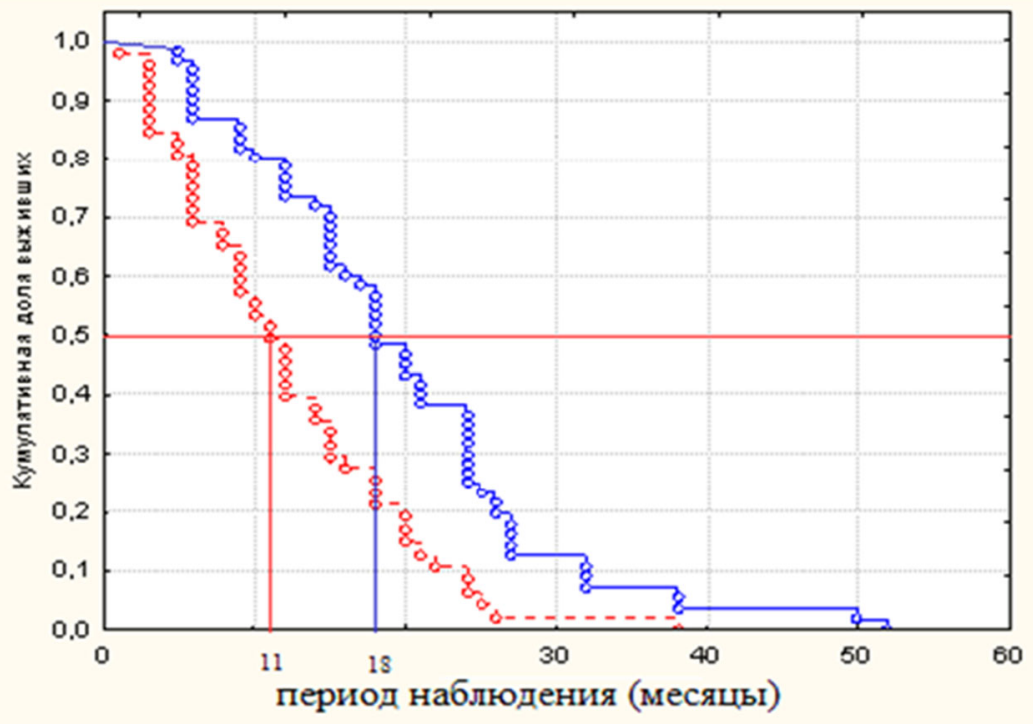

Main group

Control group 
procedure causes less surgical trauma and postoperative stress, and as a result, less immunosuppression is more justified (BenEliyahu S. et al. 1999; Sietses C. et al. 1999). One of such methods is RFA.

The results of a comparative analysis of the RFA effectiveness in the combined treatment of bilobar liver metastases of colorectal cancer (main group) with the results of using chemotherapy alone (control group) are presented in this article.

RFA is a minimally invasive method of local destruction, widely used in the combined treatment of metastatic liver cancer [4]. Gleisner A.L. et al. (2008) presented the results of a nonrandomized controlled clinical study involving 258 patients who got RFA of liver metastasis CRC. The study showed that the 3-year DFS was much higher in patients who underwent surgical intervention- $40 \%$ than that in patients after RFA-34\% $(p=0.01)$ [6]. However, according to Siperstein A.E. et al. (2007) in monitoring 243 patients and Gillams AR, Lees WR (2009), after the treatment of 309 patients with RFA, 3- and 5-year survival rate was achieved-20 and $18 \%$, respectively $[13,17]$. The authors note that the arguments of the RFA effectiveness in the treatment of liver metastasis colorectal cancer are difficult to interpret because of the obvious differences in the selection criteria of patients and therapy of the above-mentioned studies which in turn demonstrate the relevance of the work.

In case of identification of bilobar liver metastasis CRC in the main group of patients, they received chemotherapy until the stabilization of the metastatic process, which was evaluated by RECIST criteria. And after that, RFA was performed for metastatic lesions $[12,18]$.

Chemotherapy on the preoperative stage has several important purposes. According to Pozzo C., Barone C. (2008), a positive response to chemotherapy is also an important predictor of the risk of disease progression after surgery. In the event of new metastases on the background of chemotherapy, surgical treatment is not indicated [19].

To evaluate the long-term results, the OS and DFS were estimated in research groups.

Chemotherapy allowed to reach a 1-year DFS in the control group $-10.2 \%$. The values obtained agree with reference literature data $[14,15]$. Conducting RFA allowed to improve disease-free survival significantly. The patients from the main group showed 2-year DFS-3.4\%.

Overall survival was also significantly higher in the main group (log-rank test statistic 3.77, $p=0.000$ ). According to $\mathrm{Y}$. Patyutko (2005), during the surgical treatment of solitary liver bilobar metastases of CRC 4-year survival rates-35.7\%, with multiple bilobar liver metastasis OS - less than a year, the median up to 6 months was obtained [9]. RFA in the treatment of solitary bilobar liver metastases of colorectal cancer allowed to reach 4-year overall survival in $19 \%$ of patients, with multiple bilobar liver metastasis median overall survival reached 18 months.

\section{Conclusion}

The application of RFA in combination with chemotherapy in the treatment of bilobar liver metastases of colorectal cancer allows to improve the performance of DFS and overall survival significantly, compared with the group of patients who received only chemotherapy.

\section{References}

1. Vashakmadze LA, Trachtenberg AK, Chomyakov VM (2007) Cytoreductive surgery for metastatic colorectal cancer (review). Palliat Med Rehabil 1:40-50

2. Davydov MI, Axel EM (2011) Statistics malignancy in Russia and the CIS countries in 2009. Bulletin GU RCRC. Blokhin RAMN T.22.- №3(85) (ap.1):172

3. Chissov VI, Butenko AV, Vashakmadze LA et al (2010) Surgical treatment of primary and metastatic liver cancer. J Russian Cancer $5: 8-12$

4. Dolgushin BI, Patyutko YI, Sholokhov VN, Kosirev VY (2007) Radiofrequency thermoablation of liver tumors. Edited by MI Davydov. M.: Practical Medicine, pp 192

5. Gantsev Sh.Kh., Ishmuratova R.Sh., Rakhmatullina IR et al (2010) Short-term results of treatment of patients with liver metastases by radiofrequency thermoablation. Proceedings of the XVII International Congress of surgeons-hepatologists of CIS countries "Actual problems of surgical hepatology". C 262-263

6. Gleisner AL, Choti MA, Assumpcao L et al (2008) Colorectal liver metastases: recurrence and survival following hepatic resection, radiofrequency ablation, and combined resection-radiofrequency ablation. Arch Surg 143(12):1204-1212

7. The diagnosis and treatment of cancer. Clinical protocols. Edited by V.I. Chissov M .: FGBU "MNIOI them. P.A.Herzen" 2013. -599s

8. Kosirev VY, Dolgushin BI (2011) Features radiofrequency thermoablation of malignant liver tumors. Review of the literature. Med Imaging 3:24-37

9. Patyutko YI (2005) Surgical treatment of malignant liver tumors. M.: Pract Med 312

10. Adam R, Wicherts DA, de Haas RJ et al (2008) Complete pathologic response after preoperative chemotherapy for colorectal liver metastases: myth or reality? J Clin Oncol 26:1635-1641

11. Wisniewski VA, Fedorov AV, Ionkin DA et al (2010) Complications of radiofrequency thermoablation of liver malignancies. Surgery 2:18-29

12. Tyulyandin SA, Nosov DA, Perevodchikova NI (2009) The minimum clinical recommendations of the European Society for Medical Oncology (ESMO). editor Russian translation, Publishing Group RCRC. NN Blokhin RAMN.-M., pp 288

13. Siperstein AE, Berber E, Ballem N, Parikh RT (2007) Survival after radiofrequency ablation of colorectal liver metastases: 10 year experience. Ann Surg 246:559-565

14. Tanaka K, Da Shima H, Ueda M et al (2008) Role of hepatectomy in treating multiple bilobar colorectal cancer metastases. Surgery 143:259-270

15. Perevodchikova NI, Steninova MB (2014) Guidelines for chemotherapy of neoplastic diseases. Moscow: Practice.

16. Sidorov DV, Lozhkin MV, Grishin NA, Petrov LO et al. 2013 Results anatomical resection of liver metastases of colorectal cancer using the technique of water-jet dissection of the parenchyma. 
Oncology XXI century - from research into clinical practice, Materials of VIII Congress of Oncologists Russia 11-13 September 2013, SPb.: T.III. pp 998-999

17. Gillams AR, Lees WR (2009) Five year survival in 309 patients with colorectal liver metastases treated with radiofrequency ablation. Eur Radiol 19:1206-1213
18. Eisenhauer EA, Therasse P, Bogaerts J, Schwartz LH, Sargent D, Ford R, Dancey J, Arbuck S, Gwyther S, Mooney M, Rubinstein L, Shankar L, Dodd L, Kaplan R, Lacombe D, Verweij J (2009) New response evaluation criteria in solid tumours: revised RECIST guideline (version 1.1). Eur J Cancer 45:228-247

19. Pozzo C, Barone C (2008) Advances in neoadjuvant therapy for colorectal cancer liver metastases. Cancer Treat Rev 34:293-301 